\title{
HIGHER ORDER ELLIPTIC BOUNDARY VALUE PROBLEMS IN SPACES WTTH HOMOGENEOUS NORMS
}

\author{
A. J. PRYDE \\ (Received 8 April 1980) \\ Communicated by R. Vyborny
}

\begin{abstract}
We consider general boundary value problems for homogeneous elliptic partial differential operators with constant coefficients. Under natural conditions on the operators, these problems give rise to isomorphisms between the appropriate spaces with homogeneous norms. We also consider operators which are not properly elliptic and boundary systems which do not satisfy the complementing condition and determine when they give rise to left or right invertible operators. A priori inequalities and regularity results for the corresponding boundary value problems in Sobolev spaces are then readily obtained.
\end{abstract}

1980 Mathematics subject classification (Amer. Math. Soc.): 35 J 40, 35 E 20.

\section{Introduction}

In this paper we extend the results of Pryde (1980b) to general boundary value problems for elliptic operators of arbitrary order acting between spaces with homogeneous norms.

We consider partial differential operators $A=A(D)$, with symbols $A(\xi)$, of the form

$$
A(\xi)=\sum_{j=0}^{m} a_{j}\left(\xi^{\prime}\right) \xi_{n},
$$

where the $a_{j}\left(\xi^{\prime}\right)$ are positively homogeneous of order $m-j$ and are continuous for $\xi^{\prime} \neq 0$.

CCopyright Australian Mathematical Society 1981

This research was partially supported by the Natural Sciences and Engineering Research Council of Canada. 
Here, and elsewhere, $\xi=\left(\xi^{\prime}, \xi_{n}\right)=\left(\xi_{1}, \ldots, \xi_{n}\right) \in R^{n}$. As usual, $A$ is called elliptic if $A(\xi) \neq 0$ for $\xi \neq 0$.

We also consider boundary systems $B=\left(B_{j}\right), 0<j<p-1$, with symbols $\left(B_{j}(\xi)\right)$, of the form

$$
B_{j}(\xi)=\sum_{k=0}^{m_{j}} b_{j k}\left(\xi^{\prime}\right) \xi_{n}^{k}
$$

where the $b_{j k}\left(\xi^{\prime}\right)$ are positively homogeneous of order $m_{j}-k$ and are continuous for $\xi^{\prime} \neq 0$.

Spaces with homogeneous norms are discussed in detail in Pryde (1980a). Here we summarise their definitions. For $s>0, Z^{s}\left(R^{n}\right)$ is the completion of $C_{0}^{\infty}\left(R^{n}\right)$ with the norm

$$
\left[u, R^{n}\right]_{s}=\left(\int|\xi|^{2 s}|\hat{u}(\xi)|^{2} d \xi\right)^{1 / 2} .
$$

It is realised as a subspace of $\delta^{\prime}\left(R^{n}\right) / P^{s-n / 2}\left(R^{n}\right)$, the Schwartz space of tempered distributions factored by polynomials of degree $<s-n / 2$. The dual of $Z^{s}\left(R^{n}\right)$ is $Z^{-s}\left(R^{n}\right)$, a subspace of $\delta^{\prime}\left(R^{n}\right)$ when the pairing is given by an extension of the inner product in $L^{2}\left(R^{n}\right)$. Let $R_{+}^{n}=\left\{x=\left(x_{1}, \ldots, x_{n}\right) \in R^{n}\right.$ : $\left.x_{n}>0\right\}$ and $R_{-}^{n}=R^{n}-\overline{R_{+}^{n}}$. Then for arbitrary real $s$, the space of restrictions to $\Omega=R_{+}^{n}$ or $R_{-}^{n}$ of members of $Z^{s}\left(R^{n}\right)$, with the infimum norm, is complete and is denoted by $Z^{s}(\Omega)$. Relative to an extension of the inner product of $L^{2}(\Omega)$, $Z^{-s}(\Omega)$ is dual to $\dot{Z}^{s}(\Omega)$ the subspace of $Z^{s}\left(R^{n}\right)$ consisting of factor classes containing a distribution with support in $\bar{\Omega}$. For $s \geqslant 0$ and $\Omega=R^{n}, R_{+}^{n}$ or $R_{-}^{n}$, there are continuous dense embeddings $H^{s}(\Omega) \subset Z^{s}(\Omega)$ and $Z^{-s}(\Omega) \subset H^{-s}(\Omega)$, where the $H^{t}(\Omega)$ are Sobolev spaces defined in the usual way.

In Sections 2 and 6 we show how interior and boundary value problems give rise to isomorphisms

$$
\begin{aligned}
& A: Z^{s}\left(R^{n}\right) \rightarrow Z^{s-m}\left(R^{n}\right), \quad \text { and } \\
& B: Z_{\text {ker } A}^{s}\left(R_{+}^{n}\right) \rightarrow \sum_{j=0}^{p-1} Z^{s-m_{j}-(1 / 2)}\left(R^{n-1}\right),
\end{aligned}
$$

where $\operatorname{ker} A$ denotes the kernel of $A$ in the indicated space. Moreover, the relevant conditions on $A(\xi)$ and $B_{j}(\xi)$ are shown to be both necessary and sufficient. At the same time we obtain results for elliptic operators that are not properly elliptic and for boundary systems that do not satisfy the complementing condition of Agmon-Douglis-Nirenberg (1959). Indeed, we give necessary and sufficient conditions on the symbols in order that $B$ above should be left or right invertible.

Since spaces with homogeneous norms are not in general spaces of distributions, we prove in Section 3 a density theorem in order to use Fourier transform 
techniques. This result is used in Sections 4 and 5 to define the action of trace and other boundary operators on $Z_{\mathrm{ker} A}^{s}\left(R_{+}^{n}\right)$.

As a simple consequence of the previous results, we obtain in Sections 7 and 8 a priori inequalities and regularity results in Sobolev spaces.

A number of previous authors have used spaces with homogeneous norms to study elliptic boundary value problems. For example, see Pryde (1980a) for references to the work of Deny, Lions, Hormander, Aronszajn, Smith, Shamir and others. These spaces are especially suited to the study of constant coefficient operators on $R_{+}^{n}$. By well-known localization techniques, Sobolev space estimates for operators on bounded domains readily follow.

In Pryde (1981) we used these methods to study mixed boundary value problems for second order elliptic operators on a smoothly bounded domain. We obtained necessary and sufficient conditions on the coefficients of the operators in order that the problems should give rise to Fredholm operators between appropriate Sobolev spaces. We plan, in a later paper, to extend those results to the higher order case. An essential ingredient will be certain results of the present paper.

\section{Operators on $\mathbf{R}^{n}$}

Interior results for an operator $A$ of the form (1.1) between spaces with homogeneous norms take the following simple form.

THEOREM 2.1. If $A$ is of the form (1.1) and $s$ is real, the following are equivalent.

(a) $A: Z^{s}\left(R^{n}\right) \rightarrow Z^{s-m}\left(R^{n}\right)$ is left invertible.

(b) $A: Z^{s}\left(R^{n}\right) \rightarrow Z^{s-m}\left(R^{n}\right)$ is right invertible.

(c) $A$ is elliptic.

PROOF. The theorem is an immediate consequence of the following lemma, which we will also require later.

LEMMA 2.2. Let $P=\left(P_{j k}\right)$ be a $p \times r$ matrix of pseudo-differential operators $P_{j k}$, $0 \leqslant j \leqslant p-1,0 \leqslant k \leqslant r-1$, with symbols $P_{j k}(\xi)$ which are positively homogeneous of order $\lambda_{j}-\mu_{k}$ and continuous for $\xi \neq 0$. For each real $s, P$ determines $a$ bounded operator

$$
P: \prod_{k=0}^{r-1} Z^{s-r_{k}}\left(R^{n}\right) \rightarrow \prod_{j=0}^{p-1} Z^{s-\lambda}\left(R^{n}\right)
$$

for which $P$ is left (right) invertible if and only if rank $P(\xi)=r$ (respectively $p$ ) for all $\xi \neq 0$. 


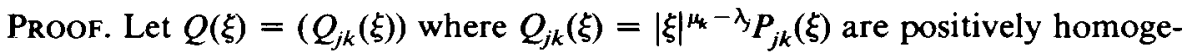
neous of order 0 and are continuous for $\xi \neq 0$. Let $J_{1}(\xi)$ and $J_{2}(\xi)$ be the diagonal matrices with entries $|\xi|^{s-\lambda_{j}}$ and $|\xi|^{\mu_{k}-s}$ respectively. These matrices are the symbols of bounded operators $Q, J_{1}$ and $J_{2}$ satisfying

$$
Q=J_{1} P J_{2}: \prod_{k=0}^{r-1} L^{2}\left(R^{n}\right) \rightarrow \prod_{j=0}^{p-1} L^{2}\left(R^{n}\right)
$$

and $J_{1}, J_{2}$ are isomorphisms between the appropriate spaces with homogeneous norms. So $P$ is left (right) invertible if and only if $Q$ is left (right) invertible, and rank $P(\xi)=\operatorname{rank} Q(\xi)$ for all $\xi \neq 0$.

Suppose $Q(\xi)$ has rank $r$ for each $\xi \neq 0$. Set $K(\xi)=\left[Q(\xi)^{*} Q(\xi)\right]^{-1} Q(\xi)^{*}, Q(\xi)^{*}$ being the conjugate transpose of $Q(\xi)$. Then $K(\xi)$ is positively homogeneous of order 0 , continuous for $\xi \neq 0$, and $K(\xi) Q(\xi)=I$. So $K(\xi)$ is the symbol of a bounded left inverse of $Q$.

Conversely, if rank $Q(\eta)<r$ for some $\eta \neq 0$, take a unit vector $c=\left(c_{k}\right)$ such that $Q(\eta) c=0$. Take $\psi \in C_{0}^{\infty}\left(R^{n}\right)$ with $\psi(\xi) \geqslant 0$ for all $\xi, \psi(\xi)=0$ for $|\xi|>1$ and $\|\psi\|_{L^{2}\left(R^{n}\right)}=1$. For $\varepsilon>0$, set $g_{\varepsilon}(\xi)=\varepsilon^{-n / 2} \psi((\xi-\eta) / \varepsilon) c$. Then $g_{\varepsilon} \in$ $\Pi_{k=0}^{r-1} L^{2}\left(R^{n}\right)$ and $\left\|g_{\varepsilon}\right\|=1$. Let $f_{\varepsilon}$ be the inverse Fourier transform of $g_{\varepsilon}$. Then $\left\|f_{e}\right\|=1$ and

$$
\begin{aligned}
\left\|Q f_{\varepsilon}\right\|^{2} & =\sum_{j=0}^{p-1} \int_{|\xi-\eta|<\varepsilon}\left|\sum_{k=0}^{r-1} Q_{j k}(\xi) \varepsilon^{-n / 2} \psi\left(\frac{\xi-\eta}{\varepsilon}\right) c_{k}\right|^{2} d \xi \\
& <\sum_{j=0}^{p-1} \sup _{|\xi-\eta|<\varepsilon}\left|\sum_{k=0}^{r-1} Q_{j k}(\xi) c_{k}\right|^{2} \rightarrow 0 \text { as } \varepsilon \rightarrow 0 .
\end{aligned}
$$

So $Q$ is not left invertible.

Since $Q^{*}$ is a pseudo-differential operator with symbol $Q(\xi)^{*}$, the remainder of the lemma follows by duality.

COROLLARY 2.3. If $A$ is an elliptic operator of the form (1.1) and $s$ is real, then

(a) $A: \dot{Z}^{s}\left(R_{+}^{n}\right) \rightarrow \dot{Z}^{s-m}\left(R_{+}^{n}\right)$ is left invertible;

(b) $A: Z^{s}\left(R_{+}^{n}\right) \rightarrow Z^{s-m}\left(R_{+}^{n}\right)$ is right invertible.

Proor. $\dot{Z}^{s}\left(R_{+}^{n}\right)$ is a closed subspace of $Z^{s}\left(R_{+}^{n}\right)$, which proves (a), and (b) follows by applying (a) to the formal adjoint of $A$ and taking duals. 


\section{A density theorem}

Our aim in this section is to prove

THEOREM 3.1. If $A$ is an elliptic operator of the form (1.1) and $s, s^{\prime}$ are real then $H_{\mathrm{ker} A}^{s^{\prime}}\left(R_{+}^{n}\right)$ is dense in $Z_{\mathrm{ker} A}^{s}\left(R_{+}^{n}\right)$. That is, the intersection of the two spaces is dense in the second.

Before proving the theorem, we define reflection operators $P$ as in Pryde (1980a). Given a positive integer $l$, let $\beta_{k}$ be the unique reals satisfying

$$
\sum_{k=1}^{l+1}\left[(-k)^{j}+(-k)^{-j}\right] \beta_{k}=1, \text { for } 0<j<l .
$$

The coefficient matrix of this system is a sum of Vandermonde matrices. Using techniques similar to those in Van der Poorten (1976), the determinant of the matrix is found to be

$$
2((l+1) !)^{-l} \prod_{j=1}^{l+1} \prod_{i=1}^{j-1}(i-j)(i j-1)
$$

which is nonzero. For $u \in C_{0}^{l}\left(\overline{R_{+}^{n}}\right)$ define $P u \in C_{0}^{l}\left(R^{n}\right)$ by

$$
(P u)(x)=\left\{\begin{array}{l}
u(x) \quad \text { if } x_{n} \geqslant 0, \\
\sum_{k=1}^{l+1}\left[u\left(x^{\prime},-k x_{n}\right)+u\left(x^{\prime},-k^{-1} x_{n}\right)\right] \beta_{k} \quad \text { if } x_{n}<0 .
\end{array}\right.
$$

As shown in Pryde (1980a), $P$ extends by continuity to a bounded operator

$$
P: Z^{s}\left(R_{+}^{n}\right) \rightarrow Z^{s}\left(R^{n}\right) \text { for } 0<s<l .
$$

Moreover, if $R: Z^{s}\left(R^{n}\right) \rightarrow Z^{s}\left(R_{+}^{n}\right)$ is the natural projection, then $R P=I$. In fact, because of our choice of $\beta_{k}$, these results are true for $-l+1<s<l$. To show this, we define an operator $P^{-}$as follows. For $u \in C_{0}^{l-1}\left(R_{-}^{n}\right)$ let $P^{-} u \in$ $C_{0}^{l-1}\left(R^{n}\right)$ be given by

$$
\left(P^{-} u\right)(x)=\left\{\begin{array}{l}
\sum_{k=1}^{l+1}\left[-k u\left(x^{\prime},-k x_{n}\right)-k^{-1} u\left(x^{\prime},-k^{-1} x_{n}\right)\right] \beta_{k} \text { if } x_{n}>0, \\
u(x) \quad \text { if } x_{n}<0 .
\end{array}\right.
$$

Then $P^{-}$extends by continuity to a bounded operator $P^{-}: Z^{-s}\left(R_{-}^{n}\right) \rightarrow Z^{-s}\left(R^{n}\right)$ when $0 \leqslant s \leqslant l-1$, and if $R^{-}: Z^{-s}\left(R^{n}\right) \rightarrow Z^{-s}\left(R_{-}^{n}\right)$ is the natural projection then $R^{-} P^{-}=I$. 
By Pryde (1980a), (3.11), each of the following sequences of operators is exact:

$$
0 \rightleftarrows \dot{Z}^{-s}\left(R_{+}^{n}\right) \underset{j}{\stackrel{i}{\rightleftarrows}} Z^{-s}\left(R^{n}\right) \underset{P^{-}}{\stackrel{R^{-}}{\rightleftarrows}} Z^{-s}\left(R_{-}^{n}\right) \rightleftarrows 0,
$$

where $0<-s \leqslant l-1, i$ is the embedding and $j=i^{-1}\left(I-P^{-} R^{-}\right)$. Taking duals we obtain exact sequences

$$
0 \rightleftarrows \dot{Z}^{s}\left(R_{-}^{n}\right) \underset{\left(P^{-}\right)^{*}}{\stackrel{\left(R^{-}\right)^{*}}{\rightleftarrows}} Z^{s}\left(R^{n}\right) \underset{P}{\stackrel{R}{\rightleftarrows}} Z^{s}\left(R_{+}^{n}\right) \rightleftarrows 0,
$$

where $R=i^{*}$ and $P=j^{*}$.

LEMMA 3.5. For $-l+1 \leqslant s \leqslant l$, the operator $P: Z^{s}\left(R_{+}^{n}\right) \rightarrow Z^{s}\left(R^{n}\right)$ is the unique bounded extension of the operator defined by (3.3) and satisfies $R P=I$.

Proof. For $s \geqslant 0$, the result is discussed above. So suppose $-l+1<s<0$. Let $u \in C_{0}^{\prime}\left(\overline{R_{+}^{n}}\right) \cap Z^{s}\left(R_{+}^{n}\right)$ and $v \in C_{0}^{\infty}\left(R^{n}\right)$ which is dense in $Z^{-s}\left(R^{n}\right)$. Then

$$
\begin{aligned}
\langle P u, v\rangle & =\left\langle j^{*} u, v\right\rangle \\
& =\langle u, j v\rangle \\
& =\left\langle u, i^{-1}\left(I-P^{-} R^{-}\right) v\right\rangle \\
& =\int_{R_{+}^{n}} u(x)\left(\bar{v}(x)-\sum_{k=1}^{++1}\left[-k \bar{v}\left(x^{\prime},-k x_{n}\right)-k^{-1} \bar{v}\left(x^{\prime},-k^{-1} x_{n}\right)\right] \beta_{k}\right) d x \\
& =\int_{R_{+}^{n}} u(x) \bar{v}(x) d x+\int_{R_{-}^{n}} \sum_{k=1}^{l+1}\left[u\left(x^{\prime},-k^{-1} x_{n}\right)+u\left(x^{\prime},-k x_{n}\right)\right] \beta_{k} \bar{v}(x) d x \\
& =\int_{R_{+}^{n}}(P u)(x) \bar{v}(x) d x,
\end{aligned}
$$

where this last $P$ is given by (3.3). Finally, since $R^{-} i=0, R P=i^{*} j^{*}=(j i)^{*}=$ $\left[i^{-1}\left(I-P^{-} R^{-}\right) i\right]^{*}=I$.

LEMMA 3.6. Let $A$ be an elliptic operator of the form (1.1), take $t \geqslant 0$, and let $P$ be a reflection operator corresponding to an integer $l \geqslant \max (m+1, t-m)$. If $u \in H^{i}\left(R^{n}\right)$ then $A^{-1} P R A u \in H^{i}\left(R^{n}\right)$.

Proof. Let $u \in H^{t}\left(R^{n}\right)$ and take $0 \leqslant s \leqslant t$. then $u \in Z^{s}\left(R^{n}\right)$ so $R A u \in$ $Z^{s-m}\left(R_{+}^{n}\right)$. By Lemma 3.5 , since $-l+1 \leqslant-m<s-m \leqslant t-m<l$, PRAu $Z^{s-m}\left(R^{n}\right)$. By Theorem 2.1, $A^{-1} P R A u \in Z^{s}\left(R^{n}\right)$. So $A^{-1} P R A u \in H^{t}\left(R^{n}\right)$. 
Proof of Theorem 3.1. Let $\boldsymbol{P}$ be a reflection operator corresponding to an integer $l$ such that $-l+1 \leqslant s \leqslant l, l \geqslant m+1$ and $l>s^{\prime}-m$. Let $u \in$ $Z_{\text {ker } A}^{s}\left(R_{+}^{n}\right)$ and take $\varphi_{i} \in C_{0}^{\infty}\left(R^{n}\right) \cap Z^{s}\left(R^{n}\right)$ with $\varphi_{i} \rightarrow P u$ in $Z^{s}\left(R^{n}\right)$. Set $\psi_{i}=$ $\varphi_{i}-A^{-1} P R A \varphi_{i}$. By Lemma 3.6, $\psi_{i} \in H^{l+m}\left(R^{n}\right) \subset H^{s^{\prime}}\left(R^{n}\right)$. But $A^{-1} P R A \varphi_{i}=$ $A^{-1} P A R \varphi_{i} \rightarrow A^{-1} P A R P u=A^{-1} P A u=0$, so $\psi_{i} \rightarrow P u$ in $Z^{s}\left(R^{n}\right)$. Finally, $A R \psi_{i}=$ $A R \varphi_{i}-A R A^{-1} P R A \varphi_{i}=A R \varphi_{i}-R A A^{-1} P R A \varphi_{i}=0$. So $R \psi_{i} \in H_{\text {ker } A}^{s^{\prime}}\left(R_{+}^{n}\right)$ and $R \psi_{i} \rightarrow u$ in $Z^{s}\left(R_{+}^{n}\right)$.

\section{The trace operator}

For $s>\frac{1}{2}$, the trace operator $\gamma_{0}$ defined by $\left(\gamma_{0} u\right)\left(x^{\prime}\right)=u\left(x^{\prime}, 0\right)$ extends to a bounded operator $\gamma_{0}: Z^{s}\left(R_{+}^{n}\right) \rightarrow Z^{s-(1 / 2)}\left(R^{n-1}\right)$. Indeed, if $u$ is smooth and $P$ is a suitable reflection operator, $\left(\gamma_{0} u\right)^{-}\left(\xi^{\prime}\right)=1 / \sqrt{2 \pi} \int_{-\infty}^{\infty}(P u)^{\wedge}\left(\xi^{\prime}, \xi_{n}\right) d \xi_{n}$. Hence

$$
\begin{aligned}
& \left\|\gamma_{0} u\right\|_{Z^{s-(1 / 2)}\left(R^{n-1}\right)}^{2}=\int_{R^{n-1}}\left|\xi^{\prime}\right|^{2 s-1}\left|\left(\gamma_{0} u\right)^{\wedge}\left(\xi^{\prime}\right)\right|^{2} d \xi^{\prime} \\
& \quad \leqslant \frac{1}{2 \pi} \int_{R^{n-1}}\left|\xi^{\prime}\right|^{2 s-1}\left(\int_{-\infty}^{\infty}\left(\left|\xi^{\prime}\right|^{2}+\xi_{n}^{2}\right)^{-s} d \xi_{n}\right)\left(\int_{-\infty}^{\infty}|\xi|^{2 s}\left|(P u)^{-}(\xi)\right|^{2} d \xi_{n}\right) d \xi^{\prime} \\
& \quad=\frac{1}{2 \pi}\left(\int_{-\infty}^{\infty}\left(1+t^{2}\right)^{-s} d t\right)\left(\int_{R^{n}}|\xi|^{2 s} \mid(P u)^{\wedge}(\xi)^{2} d \xi\right) \\
& \quad \leqslant c\|u\|_{Z^{s}\left(R_{+}^{n}\right),}^{2} \text { if } s>\frac{1}{2}
\end{aligned}
$$

In this section, we construct from $\gamma_{0}$ a bounded operator $\gamma_{0}: Z_{\text {ker } A}^{s}\left(R_{+}^{n}\right) \rightarrow$ $Z^{s-(1 / 2)}\left(R^{n-1}\right)$, for arbitrary real $s$, and $A$ an elliptic operator of the form (1.1).

For such an $A$, denote by $A^{\prime}$ the formal adjoint of $A$. So $A^{\prime}(\xi)=\overline{A(\xi)}$.

As usual, set $D_{k}=(1 / i)\left(\partial / \partial x_{k}\right), 1 \leqslant k<n$, and define $\gamma_{j}=\gamma_{0} D_{n}^{j}$.

Let $\left\{\delta_{j}: 0 \leqslant j \leqslant m-1\right\}$ be the adjoint system of boundary operators for $\left\{\gamma_{j}\right.$ : $0 \leqslant j \leqslant m-1\}$ relative to $A$. That is, the $\delta_{j}$ are the uniquely determined homogeneous boundary operators of order $m-j-1$, satisfying Green's formula

$$
(A u, v)-\left(u, A^{\prime} v\right)=\sum_{j=0}^{m-1}\left\langle\gamma_{j} u, \delta_{j} v\right\rangle \text { for } u, v \in C_{0}^{m}\left(\overline{R_{+}^{n}}\right)
$$

Here $($,$) and \langle$,$\rangle denote respectively the inner products in L^{2}\left(R_{+}^{n}\right)$ and $L^{2}\left(R^{n-1}\right)$. The $\delta_{j}$ have symbols of the form $\delta_{j}(\xi)=\sum_{k=0}^{m-j-1} d_{j k}\left(\xi^{\prime}\right) \xi_{n}^{k}$, where the $d_{j k}\left(\xi^{\prime}\right)$ are positively homogeneous of order $m-j-k-1$ and are continuous for $\xi^{\prime} \neq 0$. Since $A$ is elliptic, the $\delta_{j}$ are normal. That is, $d_{j, m-j-1}\left(\xi^{\prime}\right) \neq 0$ for $\xi^{\prime} \neq 0$. 
Now let $l$ be a positive integer. Then $\delta=\left(\delta_{m-1}, \ldots, \delta_{0}, \gamma_{0} A^{\prime}, \ldots, \gamma_{l-1} A^{\prime}\right)$ is a Dirichlet system of order $m+l$. That is, all components are normal and their orders run from 0 through $m+l-1$. It follows that

$$
0 \rightarrow \mathcal{Z}^{m+l}\left(R_{+}^{n}\right) \stackrel{\subsetneq}{\rightarrow} Z^{m+l}\left(R_{+}^{n}\right) \stackrel{\delta}{\rightarrow} \prod_{j=0}^{m+l-1} Z^{m+l-j-(1 / 2)}\left(R^{n-1}\right) \rightarrow 0
$$

is an exact sequence. Indeed $\delta=\mathscr{D} \gamma$ where $\gamma=\left(\gamma_{0}, \ldots, \gamma_{m+l-1}\right)$ and $\mathscr{D}=$ $\left(\mathscr{D}_{j k}\right), 0<j, k \leqslant m+l-1$, is a lower triangular matrix of homogeneous differential operators $\mathscr{D}_{j k}$ of orders $j-k$ acting in the boundary $R^{n-1}$. By the normality, $\mathscr{D}$ is invertible and its inverse is also a matrix of differential operators. Consequently $\mathscr{D}$ defines an automorphism on $\Pi_{k=0}^{m+l-1} Z^{m+l-k-(1 / 2)}\left(R^{n-1}\right)$. By Pryde (1980a), Theorem 4.1, sequence (4.2) with $\delta$ replaced by $\gamma$ is exact. The present result is therefore proved.

If $X^{m+l}\left(R_{+}^{n}\right)=\left\{v \in Z^{m+l}\left(R_{+}^{n}\right):\left(\delta_{m-1}, \ldots, \delta_{1}, \gamma_{0} A^{\prime}, \ldots, \gamma_{l-1} A^{\prime}\right) v=0\right\}$ then $A^{\prime}: X^{m+l}\left(R_{+}^{n}\right) \rightarrow \mathcal{Z}^{\prime}\left(R_{+}^{n}\right)$ is a bounded operator, and the following sequence is exact:

$$
0 \rightarrow Z^{m+l}\left(R_{+}^{n}\right) \underset{\subset}{\stackrel{i}{\rightarrow}} X^{m+l}\left(R_{+}^{n}\right) \stackrel{\delta_{0}}{\rightarrow} Z^{l+(1 / 2)}\left(R^{n-1}\right) \rightarrow 0
$$

So the rows in the following diagram are exact and we define $\gamma_{0}: Z_{\text {ker } A}^{-l}\left(R_{+}^{n}\right)$ $\rightarrow Z^{-l-(1 / 2)}\left(R^{n-1}\right)$ to be the unique bounded operator which makes the diagram commute.

$$
\begin{aligned}
& \begin{array}{c}
0 \rightarrow Z_{\mathrm{ker} A}^{-l}\left(R_{+}^{n}\right) \\
\downarrow \gamma_{0}
\end{array} \quad \stackrel{\subset}{\rightarrow} \quad \begin{array}{ccccc}
Z^{-l}\left(R_{+}^{n}\right) & \rightarrow & Z^{-l-m}\left(R_{+}^{n}\right) & \rightarrow & 0
\end{array} \\
& 0 \rightarrow Z^{-l-(1 / 2)}\left(R^{n-1}\right) \underset{-\delta_{0}^{*}}{\rightarrow} X^{m+l}\left(R_{+}^{n}\right)^{*} \quad \underset{i^{*}}{\rightarrow} Z^{-l-m}\left(R_{+}^{n}\right) \quad \rightarrow \quad 0 .
\end{aligned}
$$

For $s>-l$ we define $\gamma_{0}: Z_{\text {ker } A}^{s}\left(R_{+}^{n}\right) \rightarrow Z^{s-(1 / 2)}\left(R^{n-1}\right)$ to be the composite of the following sequence of bounded operators

$$
Z_{\mathrm{ker} A}^{s}\left(R_{+}^{n}\right) \stackrel{\left|\nabla^{\prime}\right|^{s+l}}{\rightarrow} Z_{\mathrm{ker} A}^{-l}\left(R_{+}^{n}\right) \stackrel{\gamma_{0}}{\rightarrow} Z^{-l-(1 / 2)}\left(R^{n-1}\right) \stackrel{\left|\nabla^{\prime}\right|^{-s-l}}{\rightarrow} Z^{s-(1 / 2)}\left(R^{n-1}\right)
$$

THEOREM 4.6. If $A$ is an elliptic operator of the form (1.1) and $s$ is real, $\gamma_{0}$ : $Z_{\mathrm{ker} A}^{s}\left(R_{+}^{n}\right) \rightarrow Z^{s-(1 / 2)}\left(R^{n-1}\right)$ is the unique bounded extension of the mapping $u \rightarrow u \mid R^{n-1}$ on $Z_{\text {ker } A}^{s}\left(R_{+}^{n}\right) \cap C^{0}\left(\overline{R_{+}^{n}}\right)$.

Proof. First take $s=-l$. Let $u \in Z_{\text {ker } A}^{-l}\left(R_{+}^{n}\right) \cap C^{m}\left(\overline{R_{+}^{n}}\right)$ and set $g=u \mid R^{n-1}$. Let $\varphi \in C_{0}^{\infty}\left(R^{n-1}\right) \cap Z^{l+(1 / 2)}\left(R^{n-1}\right)$ which is dense in $Z^{l+(1 / 2)}\left(R^{n-1}\right)$, the dual of $Z^{-l-(1 / 2)}\left(R^{n-1}\right)$. We show $\left\langle\gamma_{0} u, \varphi\right\rangle=\langle g, \varphi\rangle$. Since $\delta$ is a Dirichlet system, there exists $v \in H^{s^{\prime}}\left(R_{+}^{n}\right)$, where $s^{\prime}$ can be taken arbitrarily large, such that $\delta v=(0, \ldots, 0, \varphi, 0, \ldots, 0)$ where $\varphi$ occupies the position corresponding to $\delta_{0}$. 
If $s^{\prime} \geqslant m+l$ then $v \in X^{m+l}\left(R_{+}^{n}\right)$ and $u$ and $v$ satisfy Green's formula (4.1). So

$$
\begin{aligned}
\left\langle\gamma_{0} u, \varphi\right\rangle & =\left\langle-\left(\delta^{*}\right)^{-1}\left(A^{\prime}\right)^{*} u, \delta_{0} v\right\rangle \\
& =-\left\langle u, A^{\prime} v\right\rangle \\
& =-(A u, v)+\sum_{j=0}^{m-1}\left\langle\gamma_{j} u, \delta_{j} v\right\rangle \\
& =\left\langle g, \delta_{0} v\right\rangle \\
& =\langle g, \varphi\rangle .
\end{aligned}
$$

So $\gamma_{0} u=g$ for such $u$. However, $H^{s^{\prime \prime}}\left(R_{+}^{n}\right)$ is dense in $Z_{\text {ker } A}^{-l}\left(R_{+}^{n}\right)$ (Theorem 3.1) and $H^{s^{\prime \prime}}\left(R_{+}^{n}\right) \subset C^{m}\left(\overline{R_{+}^{n}}\right)$ if $s^{\prime \prime}>n / 2+m$. Hence $C^{m}\left(\overline{R_{+}^{n}}\right)$ is dense in $Z_{\text {ker } A}^{-l}\left(R_{+}^{n}\right)$ and $\gamma_{0}$ is uniquely determined as claimed. The result for $s>-l$ now follows.

\section{Other boundary operators}

Let $A$ be an elliptic operator of the form (1.1) and $B$ a boundary system of the form (1.2). By Theorem 4.6, for each real $s, B$ determines a bounded operator

$$
B: Z_{\mathrm{ker} A}^{s}\left(R_{+}^{n}\right) \rightarrow \prod_{j=0}^{p-1} Z^{s-m-(1 / 2)}\left(R^{n-1}\right) .
$$

We proceed now to represent $B u$ in the form $\mathscr{B} \gamma u$ where $B$ is a pseudodifferential operator acting in the boundary, and $\gamma u$ is the Cauchy data of $u$.

For each $\xi^{\prime} \neq 0, A\left(\xi^{\prime}, z\right)$ has $r\left(\xi^{\prime}\right)$ roots $z_{k}\left(\xi^{\prime}\right), 0<k<r\left(\xi^{\prime}\right)-1$, with positive imaginary part. They are not necessarily all distinct. Set $A\left(\xi^{\prime}, z\right)=$ $A^{+}\left(\xi^{\prime}, z\right) A^{-}\left(\xi^{\prime}, z\right)$ where $A^{+}\left(\xi^{\prime}, z\right)=\Pi_{k}\left(z-z_{k}\left(\xi^{\prime}\right)\right)$ and let $r=r(A)=$ $\max \left\{r\left(\xi^{\prime}\right): \xi^{\prime} \neq 0\right\}$.

By the continuity of $A(\xi)$, if $n>2$ then $r\left(\xi^{\prime}\right)=r$ for all $\xi^{\prime} \neq 0$. Similarly, if $n=2$ then $r\left(\xi^{\prime}\right)$ takes at most two values, one for positive $\xi^{\prime}$ and one for negative $\xi^{\prime}$. Recall that if $A(\xi)$ is an even order polynomial, it is called properly elliptic if $r\left(\xi^{\prime}\right)=m / 2$ for all $\xi^{\prime} \neq 0$.

Let $\mathscr{B}\left(\xi^{\prime}\right)=\left(\mathscr{B}_{j k}\left(\xi^{\prime}\right)\right)$ be the $p \times r$ matrix given by

$$
B_{j}\left(\xi^{\prime}, z\right) \equiv \sum_{k=0}^{r-1} \mathscr{B}_{j k}\left(\xi^{\prime}\right) z^{k} \quad\left(\bmod A^{+}\left(\xi^{\prime}, z\right)\right),
$$

for $0<j<p-1$, with $\mathscr{B}_{j k}\left(\xi^{\prime}\right)=0$ if $r\left(\xi^{\prime}\right)<k<r-1$. So $\mathscr{B}_{j k}\left(\xi^{\prime}\right)$ is positively homogeneous of order $m_{j}-k$ and is continuous for $\xi^{\prime} \neq 0$. Hence $\mathscr{B}\left(\xi^{\prime}\right)$ is the symbol of a bounded operator

$$
\mathscr{B}: \prod_{k=0}^{r-1} Z^{s-k-(1 / 2)}\left(R^{n-1}\right) \rightarrow \prod_{j=0}^{p-1} Z^{s-m-(1 / 2)}\left(R^{n-1}\right) .
$$


Recall that if $A$ is properly elliptic and $p=r=m / 2$ then $B$ satisfies the complementing condition (or the Shapiro-Lopatinski condition) with respect to $A$ if $\mathscr{B}\left(\xi^{\prime}\right)$ is non-singular for $\xi^{\prime} \neq 0$.

Finally, set $\gamma=\left(\gamma_{0}, \ldots, \gamma_{r-1}\right)$ which by Theorem 4.6 extends uniquely to a bounded operator

$$
\gamma: Z_{\mathrm{ker} A}^{s}\left(R_{+}^{n}\right) \rightarrow \prod_{k=0}^{r-1} Z^{s-k-(1 / 2)}\left(R^{n-1}\right) .
$$

THEOREM 5.4. Let $A$ be an elliptic operator of the form (1.1), $B$ a boundary system of the form (1.2) and $s$ real. Then $B u=\mathscr{B} \gamma u$ for all $u \in Z_{\mathrm{ker} A}^{s}\left(R_{+}^{n}\right)$.

Proof. By the density theorem (3.1), it suffices to prove the result for $u \in H_{\text {ker } A}^{l}\left(R_{+}^{n}\right)$ for suitably large $l$. Now

$$
B_{j}\left(\xi^{\prime}, z\right)=\sum_{k=0}^{r-1} \mathscr{B}_{j k}\left(\xi^{\prime}\right) z^{k}+Q_{j}\left(\xi^{\prime}, z\right) A^{+}\left(\xi^{\prime}, z\right)
$$

where $Q_{j}\left(\xi^{\prime}, z\right)$ is a polynomial in $z$ which is positively homogeneous in $\left(\xi^{\prime}, z\right)$ of order $m_{j}-r\left(\xi^{\prime}\right)$, and is continuous for $\xi^{\prime} \neq 0$. So

$$
B_{j} u=\sum_{k=0}^{r-1} \mathscr{B}_{j k} \gamma_{k} u+\gamma_{0} Q_{j} A^{+} P u
$$

for a suitable reflection operator $P$. It suffices to prove that $Q_{j} A^{+} P u \in$ $\stackrel{\circ}{H}^{l-m}\left(R_{-}^{n}\right)\left(\subset \operatorname{ker} \gamma_{0}\right)$.

Now $A P u \in L^{2}\left(R_{-}^{n}\right)$ and by the Paley-Wiener theorem, for almost all $\xi^{\prime} \neq 0$, the Fourier transform $(A P u)^{\wedge}\left(\xi^{\prime}, z\right)$ is analytic in $\operatorname{Im} z>0$ and

$$
\sup _{\eta>0} \int_{-\infty}^{\infty}\left|A\left(\xi^{\prime}, \mu+i \eta\right)(P u)^{\wedge}\left(\xi^{\prime}, \mu+i \eta\right)\right|^{2} d \mu<\infty .
$$

It follows that $\left(A^{+} P u\right)^{-}\left(\xi^{\prime}, z\right)$ is analytic in $\operatorname{Im} z>0$ and (5.5) remains valid with $A$ replaced by $A^{+}$. So for almost all $\xi^{\prime} \neq 0$, the partial Fourier transform $\left(A^{+} P u\right)^{\sim}\left(\xi^{\prime},-\right) \in L^{2}\left(R_{-}^{1}\right) \cap H^{l-r(\xi)}\left(R^{1}\right)=\dot{H}^{l-r(\xi)}\left(R_{-}^{1}\right)$. Hence $Q_{j} A^{+} P u \in$ $\dot{H}^{l-m}\left(R_{-}^{n}\right)$ as required.

\section{Boundary value problems}

In proving the theorem of this section we construct left and right inverses of boundary operators. The main step is the following:

LemMa 6.1. Let $\Gamma$ be a positively oriented simple closed contour in the complex plane on and within which the function $f(z)$ is analytic except for a finite number of poles, interior to $\Gamma$, whose orders total $r$. If $r>0$ there exist unique polynomials 
$p_{j}(z)$ for $0 \leqslant j \leqslant r-1$, of order at most $r-1$, independent of the particular contour enclosing the given poles, and satisfying

$$
\int_{\Gamma} f(z) z^{j} p_{j^{\prime}}(z) d z=\delta_{i j^{\prime}} \text { for } 0<j, j^{\prime}<r-1 .
$$

Moreover, if $f(z)=f_{1}(z) f_{2}(z)$ where $f_{1}(z)$ is analytic on and within $\Gamma$ then

$$
f_{2}(z)\left[u(z)-\sum_{j=0}^{r-1} f_{1}(z) p_{j}(z) \int_{\Gamma} f_{2}(\zeta) \zeta^{j} u(\zeta) d \zeta\right]
$$

is analytic on and within $\Gamma$, apart from removable singularities, whenever $u(z)$ has that property.

Proof. Consider the matrix $\left(q_{j k}\right)$ where $q_{j k}=\int_{\Gamma} f(z) z^{j+k} d z$ for $0<j, k<$ $r-1$. If this matrix is singular, there exist complex numbers $\alpha_{k}$, not all zero, such that $\sum_{k=0}^{r-1} q_{j k} \alpha_{k}=0$ for each $j$. Setting $g(z)=\sum_{k=0}^{r-1} \alpha_{k} z^{k}$, it follows that $\int_{\Gamma} f(z) g(z) h(z) d z=0$ for all polynomials $h(z)$ of order at most $r-1$. Within $\Gamma$, $f(z) g(z)$ has poles whose orders total at least 1 and at most $r$. So we can choose a polynomial $h(z)$ of order $r-1$ such that $f(z) g(z) h(z)$ has precisely one pole within $\Gamma$ and that of order 1 . But then $\int_{\Gamma} f(z) g(z) h(z) d z \neq 0$, contradicting a previous conclusion.

So $\left(q_{j k}\right)$ is non-singular and there exist unique complex numbers $p_{k j}$, satisfying $\sum_{k=0}^{r-1} q_{j k} p_{k j^{\prime}}=\delta_{i j^{\prime}}, 0 \leqslant j, j^{\prime} \leqslant r-1$. Setting $p_{j^{\prime}}(z)=\sum_{k=0}^{r-1} p_{k j^{\prime}} z^{k}$ we obtain (6.2). The uniqueness also follows.

Now suppose the distinct poles of $f(z)$ and $f_{2}(z)$ within $\Gamma$ are $z_{0} \ldots, z_{q}$ with orders $m_{0}, \ldots, m_{q}$ where $m_{0}+\cdots+m_{q}=r$. Using the notation $\operatorname{Res}[f(z), \zeta]$ to denote the residue of a meromorphic function $f(z)$ at $\zeta$, we obtain from (6.2)

$$
2 \pi i \sum_{l=0}^{q} \operatorname{Res}\left[f_{1}(z) f_{2}(z) z^{j} p_{j^{\prime}}(z), z_{l}\right]=\delta_{i j^{\prime}}
$$

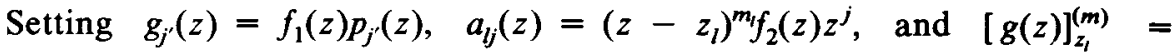
$d^{m} g\left(z_{l}\right) / d z^{m}$ we obtain

$$
\begin{aligned}
\delta_{i j^{\prime}} & =2 \pi i \sum_{l=0}^{q} \operatorname{Res}\left[f_{2}(z) z^{j} g_{j^{\prime}}(z), z_{l}\right] \\
& =2 \pi i \sum_{l=0}^{q} \frac{1}{\left(m_{l}-1\right) !}\left[a_{l j}(z) g_{j^{\prime}}(z)\right]_{z_{l}}^{\left(m_{j}-1\right)} \\
& =2 \pi i \sum_{l=0}^{q} \sum_{s=0}^{m_{l}-1}\left(\begin{array}{c}
m_{l}-1 \\
s
\end{array}\right) a_{l j}^{\left(m_{l}-1-s\right)}\left(z_{l}\right) g_{j^{\prime}}^{(s)}\left(z_{l}\right) .
\end{aligned}
$$

This can be considered as a matrix equation, and since the index $(l, s)$ runs through the $r$ values given by $0 \leqslant l \leqslant q, 0 \leqslant s \leqslant m-1$, the product can be 
reversed to give

$$
\delta_{l \prime} \delta_{s s^{\prime}}=2 \pi i \sum_{j=0}^{r-1} g_{j}^{\left(s^{\prime}\right)}\left(z_{l^{\prime}}\right) \frac{1}{\left(m_{l}-1\right) !}\left(\begin{array}{c}
m_{l}-1 \\
s
\end{array}\right) a^{\left(m_{l}-1-s\right)}\left(z_{l}\right)
$$

for $0 \leqslant l^{\prime} \leqslant q$ and $0 \leqslant s^{\prime} \leqslant m_{l},-1$. Multiplying by $u^{(s)}\left(z_{l}\right)$ and summing over $l$ and $s$ we obtain

$$
\begin{aligned}
u^{\left(s^{\prime}\right)}\left(z_{l^{\prime}}\right) & =2 \pi i \sum_{l=0}^{q} \sum_{s=0}^{m_{l}-1} \sum_{j=0}^{r-1} g_{j}^{\left(s^{\prime}\right)}\left(z_{l^{\prime}}\right) \frac{1}{\left(m_{l}-1\right) !}\left(\begin{array}{c}
m_{l}-1 \\
s
\end{array}\right) u^{(s)}\left(z_{l}\right) a_{j^{\prime}}^{\left(m_{l}-1-s\right)}\left(z_{l}\right) \\
& =2 \pi i \sum_{j=0}^{r-1} g_{j}^{\left(s^{\prime}\right)}\left(z_{l^{\prime}}\right) \sum_{l=0}^{q} \operatorname{Res}\left[u(z) f_{2}(z) z^{j}, z_{l}\right] \\
& =\sum_{j=0}^{r-1} g_{j}^{\left(s^{\prime}\right)}\left(z_{l^{\prime}}\right) \int_{\Gamma} u(\zeta) f_{2}(\zeta) \zeta^{j} d \zeta .
\end{aligned}
$$

Hence the singularities on and within $\Gamma$ of expression (6.3) are removable as claimed.

LEMMA 6.4. Suppose $f \in L^{2}\left(R_{-}^{1}\right)^{\wedge}, g$ is a function satisfying $|g(z)|>c>0$ outside some compact set $K$ in $\operatorname{Im} z>0$, and $h=f / g$ is analytic in $\operatorname{Im} z>0$. Then $h \in L^{2}\left(R_{-}^{1}\right)^{\text {. }}$.

Proof. We have only to show that $\sup _{\eta>0} \int_{-\infty}^{\infty}|h(\xi+i \eta)|^{2} d \xi<\infty$. Take $R>r>0$ with $K \subset K_{1}=\{z=\xi+i \eta:-R \leqslant \xi \leqslant R, r<\eta \leqslant R\}$ and set $S=(0, r) \cup(R, \infty)$. Then since $f \in L^{2}\left(R_{-}^{1}\right)^{\wedge}$,

$$
\sup _{\eta \in S} \int_{-\infty}^{\infty}|h(\xi+i \eta)|^{2} d \xi<\frac{1}{c^{2}} \sup _{\eta>0} \int_{-\infty}^{\infty}|f(\xi+i \eta)|^{2} d \xi<\infty ;
$$

and

$$
\begin{aligned}
\sup _{r<\eta<R} \int_{-\infty}^{\infty}|h(\xi+i \eta)|^{2} d \xi & =\sup _{r<\eta<R}\left(\int_{|\xi|>R}|h(\xi+i \eta)|^{2} d \xi+\int_{-R}^{R}|h(\xi+i \eta)|^{2} d \xi\right) \\
& <\frac{1}{c^{2}} \sup _{\eta>0} \int_{-\infty}^{\infty}|f(\xi+i \eta)|^{2} d \xi+2 R \sup _{z \in K_{1}}|h(z)|^{2} \\
& <\infty .
\end{aligned}
$$

We come now to our main result.

THEOREM 6.5. Let $A$ be an elliptic operator of the form (1.1), B a boundary system of the form (1.2) and s real. Then $B: Z_{\text {ker } A}^{s}\left(R_{+}^{n}\right) \rightarrow \prod_{j=0}^{p-1} Z^{s-m_{j}-(1 / 2)}\left(R^{n-1}\right)$ is left (right) invertible if and only if rank $\mathscr{B}\left(\xi^{\prime}\right)=r\left(\xi^{\prime}\right)$ (respectively $p$ ) for all $\xi^{\prime} \neq 0$. 
Proof. Let $h$ be a suitably large integer and for each unit vector $\eta^{\prime} \in R^{n-1}$ set $f\left(\eta^{\prime}, z\right)=(z+i)^{-h} A\left(\eta^{\prime}, z\right)^{-1}$. Let $\Gamma$ be a positively oriented simple closed contour in $\operatorname{Im} z>0$ which encloses all the roots of $A\left(\eta^{\prime}, z\right)$ in $\operatorname{Im} z>0$. For $0<j<r\left(\eta^{\prime}\right)-1$, let $p_{j}\left(\eta^{\prime}, z\right)$ be the corresponding polynomials in $z$ given by Lemma 6.1. Since $A\left(\eta^{\prime}, z\right)$ is continuous in $\eta^{\prime}$, so too are the $p_{j}\left(\eta^{\prime}, z\right)$. For $r\left(\eta^{\prime}\right)<j<r-1$, let $p_{j}\left(\eta^{\prime}, z\right)=0, r$ again denoting $\max \left\{r\left(\xi^{\prime}\right): \xi^{\prime} \neq 0\right\}$.

We define a bounded operator $Q: \prod_{j=0}^{r-1} Z^{s-j-(1 / 2)}\left(R^{n-1}\right) \rightarrow Z^{s}\left(R^{n}\right)$ to be used in constructing inverses, as follows. Let $g=\left(g_{0}, \ldots, g_{r-1}\right) \in$ $\Pi_{j=0}^{r-1} Z^{s-j-(1 / 2)}\left(R^{n-1}\right) \cap C_{0}^{\infty}\left(R^{n-1}\right)$, and set

$$
\hat{f}(\xi)=(2 \pi)^{1 / 2}|\xi|^{s}\left|\xi^{\prime}\right|^{h+m-1}\left(\xi_{n}+i\left|\xi^{\prime}\right|\right)^{-h} A(\xi)^{-1} \sum_{j=0}^{r-1}\left|\xi^{\prime}\right|^{-j} p_{j}\left(\frac{\xi^{\prime}}{\left|\xi^{\prime}\right|}, \frac{\xi_{n}}{\left|\xi^{\prime}\right|}\right) \hat{g}_{j}\left(\xi^{\prime}\right) .
$$

Then $f \in L^{2}\left(R^{n}\right)$ and $\|f\|_{L^{2}\left(R^{n}\right)} \leqslant c\|g\|$, where $c$ is independent of $g$. Indeed,

$$
\begin{aligned}
\|f\|_{L^{2}\left(R^{n}\right)}^{2} & <c\left\|\left|\xi^{\prime}\right|^{h+m-1}|\xi|^{s-h-m} \sum_{j=0}^{r-1}\left|\xi^{\prime}\right|^{-j} p_{j}\left(\frac{\xi^{\prime}}{\left|\xi^{\prime}\right|}, \frac{\xi_{n}}{\left|\xi^{\prime}\right|}\right) \hat{g}_{j}\left(\xi^{\prime}\right)\right\|_{L^{2}\left(R^{n}\right)}^{2} \\
& <c \sum_{j=0}^{r-1} \int_{R^{n}}\left|\xi^{\prime}\right|^{2 h+2 m-2-2 j}\left(\left|\xi^{\prime}\right|^{2}+\xi_{n}^{2}\right)^{s-h-m}\left|p_{j}\left(\frac{\xi^{\prime}}{\left|\xi^{\prime}\right|}, \frac{\xi_{n}}{\left|\xi^{\prime}\right|}\right)\right|^{2}\left|\hat{g}_{j}\left(\xi^{\prime}\right)\right|^{2} d \xi \\
& =c \sum_{j=0}^{r-1} \int_{R^{n-1}}\left|\xi^{\prime}\right|^{2 s-2 j-1}\left|\hat{g}_{j}\left(\xi^{\prime}\right)\right|^{2} \int_{-\infty}^{\infty}\left(1+t^{2}\right)^{s-h-m}\left|p_{j}\left(\frac{\xi^{\prime}}{\left|\xi^{\prime}\right|}, t\right)\right|^{2} d t d \xi^{\prime} \\
& <c \sum_{j=1}^{r-1} \int_{R^{n-1}}\left|\xi^{\prime}\right|^{2 s-2 j-1}\left|\hat{g}_{j}\left(\xi^{\prime}\right)\right|^{2} d \xi^{\prime}, \quad \text { if } h>s-m+r-\frac{1}{2}, \\
& =c\|g\|^{2} .
\end{aligned}
$$

Defining $Q g=|\nabla|^{-s} f$ we conclude $Q g \in Z^{s}\left(R^{n}\right)$ and $\|Q g\|_{Z^{\prime}\left(R^{n}\right)}=\|f\|_{L^{2}\left(R^{n}\right)}<$ $c\|g\|$. Extending $Q$ by continuity, we obtain a bounded operator as indicated.

With $g$ as before, we now show $R A Q g=0$. For this let $\left(D_{n}+i\left|\nabla^{\prime}\right|\right)^{s}$, as defined in Pryde (1980a), be the pseudo-differential operator with symbol $\left(\xi_{n}+i\left|\xi^{\prime}\right|\right)^{s}$. The complex powers $\zeta^{s}$ are determined by taking $-\pi<\arg \zeta<\pi$. Now set $w=\left(D_{n}+i\left|\nabla^{\prime}\right|\right)^{s-m} A Q g$. Then $w \in L^{2}\left(R^{n}\right)$ and

$$
\hat{w}(\xi)=(2 \pi)^{1 / 2}\left|\xi^{\prime}\right|^{h+m-1}\left(\xi_{n}+i\left|\xi^{\prime}\right|\right)^{s-m-h} \sum_{j=0}^{r-1}\left|\xi^{\prime}\right|^{-j} p_{j}\left(\frac{\xi^{\prime}}{\left|\xi^{\prime}\right|}, \frac{\xi_{n}}{\left|\xi^{\prime}\right|}\right) \hat{g}_{j}\left(\xi^{\prime}\right)
$$

It follows that

$$
\text { for each } \xi^{\prime} \neq 0, \hat{w}\left(\xi^{\prime}, z\right) \text { is analytic in } \operatorname{Im} z>0 \text {, and }
$$
$\sup \left\|\hat{w}\left(\xi^{\prime}, \xi_{n}+i \eta\right)\right\|_{L^{2}\left(R^{n}\right)}<\infty$. $\eta>0$ 
Indeed, the analyticity is obvious. Further $\left|p_{j}\left(\xi^{\prime}, z\right)\right|^{2}<c\left(1+|z|^{2}\right)^{r-1}$ for all $z, j$ and unit vectors $\xi^{\prime}$. Hence for $\eta>0$,

$$
\begin{aligned}
&\left|\hat{w}\left(\xi^{\prime}, \xi_{n}+i \eta\right)\right|^{2} \leqslant c\left|\xi^{\prime}\right|^{2 h+2 m-2}\left(\xi_{n}^{2}+\left(\eta+\left|\xi^{\prime}\right|\right)^{2}\right)^{s-m-h}\left(1+\frac{\xi_{n}^{2}+\eta^{2}}{\left|\xi^{\prime}\right|^{2}}\right)^{r-1} \\
& \cdot \sum_{j=0}^{r-1}\left|\xi^{\prime}\right|^{-2 j}\left|g_{j}\left(\xi^{\prime}\right)\right|^{2} \\
& \leqslant c\left|\xi^{\prime}\right|^{2 h+2 m-2 r}\left(\xi_{n}^{2}+\left(\eta+\left|\xi^{\prime}\right|\right)^{2}\right)^{s-m-h+r-1} \sum_{j=0}^{r-1}\left|\xi^{\prime}\right|^{-2 j}\left|\hat{g}_{j}\left(\xi^{\prime}\right)\right|^{2} \\
&<c\left|\xi^{\prime}\right|^{2 h+2 m-2 r}|\xi|^{2 s-2 m-2 h+2 r-2} \sum_{j=0}^{r-1}\left|\xi^{\prime}\right|^{-2 j}\left|\hat{g}_{j}\left(\xi^{\prime}\right)\right|^{2},
\end{aligned}
$$

provided $h>s-m+r-1$. This last expression is integrable if $h>s-m+r$ $-\frac{1}{2}$, in which case (6.6) is proved.

By (6.6) $w \in L^{2}\left(R_{-}^{n}\right)$. But $\left(D_{n}+i\left|\nabla^{\prime}\right|\right)^{s-m}: \dot{Z}^{s-m}\left(R_{-}^{n}\right) \rightarrow L^{2}\left(R_{-}^{n}\right)$ is an isomorphism. Hence $A Q g \in \dot{Z}^{s-m}\left(R_{-}^{n}\right)$ and $R A Q g=0$.

It follows that we have a bounded operator

$$
R Q: \prod_{k=0}^{r-1} Z^{s-k-(1 / 2)}\left(R^{n-1}\right) \rightarrow Z_{\mathrm{ker} A}^{s}\left(R_{+}^{n}\right)
$$

Suppose now that rank $\mathscr{B}\left(\xi^{\prime}\right)=p$ for all $\xi^{\prime} \neq 0$. As in the proof of Lemma 2.2, $\mathscr{B}\left(\xi^{\prime}\right)=J_{1}\left(\xi^{\prime}\right) \mathscr{B}\left(\xi^{\prime} /\left|\xi^{\prime}\right|\right) J_{2}\left(\xi^{\prime}\right)$ where $J_{1}\left(\xi^{\prime}\right)=\operatorname{diag}\left(\left|\xi^{\prime}\right|^{m}\right)$ and $J_{2}\left(\xi^{\prime}\right)=$ $\operatorname{diag}\left(\left|\xi^{\prime}\right|^{-k}\right)$. Set

$$
\mathscr{B}^{\prime}\left(\xi^{\prime}\right)=\left(\mathscr{B}_{k j}^{\prime}\left(\xi^{\prime}\right)\right)=J_{2}\left(\xi^{\prime}\right)^{-1} \mathscr{B}\left(\frac{\xi^{\prime}}{\left|\xi^{\prime}\right|}\right)^{*}\left[\mathscr{B}\left(\frac{\xi^{\prime}}{\left|\xi^{\prime}\right|}\right) \mathscr{B}\left(\frac{\xi^{\prime}}{\left|\xi^{\prime}\right|}\right)^{*}\right]^{-1} J_{1}\left(\xi^{\prime}\right)^{-1}
$$

So for $0<k<r-1,0<j<p-1, \mathscr{B}_{k j}^{\prime}\left(\xi^{\prime}\right)$ is positively homogeneous of order $k-m_{j}$ and is continuous for $\xi^{\prime} \neq 0$. Also $\mathscr{B}\left(\xi^{\prime}\right) \mathscr{B}^{\prime}\left(\xi^{\prime}\right)=I$ and

$$
B^{\prime}=R Q \mathscr{B}^{\prime}: \prod_{j=0}^{p-1} Z^{s-m-(1 / 2)}\left(R^{n-1}\right) \rightarrow Z_{\mathrm{ker} A}^{s}\left(R_{+}^{n}\right)
$$

is a bounded operator. We show that $B B^{\prime}=I$.

For this let

$$
g=\left(g_{0}, \ldots, g_{p-1}\right) \in \prod_{j=0}^{p-1} Z^{s-m_{j}-(1 / 2)}\left(R^{n-1}\right) \cap C_{0}^{\infty}\left(R^{n-1}\right)
$$


and set $g^{\prime}=B B^{\prime} g$. It suffices to show that $g^{\prime}=g$. By Theorem 5.4, $B=\mathscr{B} \gamma$ and so $g^{\prime}=\mathscr{B} \gamma R Q Q^{\prime} g$. Hence

$$
\begin{aligned}
& g_{j^{\prime}}^{\prime}\left(\xi^{\prime}\right)=\sum_{k^{\prime}=0}^{r-1} \mathscr{B}_{j^{\prime} k^{\prime}}\left(\xi^{\prime}\right)(2 \pi)^{-1 / 2} \int_{-\infty}^{\infty} \xi_{n}^{k^{\prime}}\left(Q^{\mathscr{B}^{\prime}} g\right)^{\wedge}\left(\xi^{\prime}, \xi_{n}\right) d \xi_{n} \\
& =\sum_{k^{\prime}=0}^{r-1} \mathscr{B}_{j^{\prime} k^{\prime}}\left(\xi^{\prime}\right) \int_{-\infty}^{\infty} \xi_{n}^{k^{\prime}}\left|\xi^{\prime}\right|^{h+m-1}\left(\xi_{n}+i\left|\xi^{\prime}\right|\right)^{-h} A(\xi)^{-1} \\
& \cdot \sum_{k=0}^{r-1}\left|\xi^{\prime}\right|^{-k} p_{k}\left(\frac{\xi^{\prime}}{\left|\xi^{\prime}\right|}, \frac{\xi_{n}}{\left|\xi^{\prime}\right|}\right)_{j=1}^{p-1} \mathscr{B}_{k j}^{\prime}\left(\xi^{\prime}\right) \hat{g}_{j}\left(\xi^{\prime}\right) d \xi_{n} \\
& =\sum_{k^{\prime}=0}^{r\left(\xi^{\prime}\right)-1} \mathscr{B}_{j^{\prime} k^{\prime}}\left(\xi^{\prime}\right)\left|\xi^{\prime}\right|^{k^{\prime}} \sum_{j=0}^{p-1} \hat{g}_{j}\left(\xi^{\prime}\right) \sum_{k=0}^{r\left(\xi^{\prime}\right)-1} \mathscr{P}_{k j}^{\prime}\left(\xi^{\prime}\right)\left|\xi^{\prime}\right|^{-k} \\
& \cdot \int_{-\infty}^{\infty}(t+i)^{-h} A\left(\frac{\xi^{\prime}}{\left|\xi^{\prime}\right|}, t\right)^{-1} t^{k^{\prime}} p_{k}\left(\frac{\xi^{\prime}}{\left|\xi^{\prime}\right|}, t\right) d t \\
& \text { (since } \mathscr{B}_{j^{\prime} k^{\prime}}\left(\xi^{\prime}\right)=0 \text { for } r\left(\xi^{\prime}\right)<k<r-1 \text { ) } \\
& =\sum_{k^{\prime}=0}^{r\left(\xi^{\prime}\right)-1} \mathscr{B}_{j^{\prime} k^{\prime}}\left(\xi^{\prime}\right)\left|\xi^{\prime}\right|^{k^{\prime}} \sum_{j=0}^{p-1} \hat{g}_{j}\left(\xi^{\prime}\right) \sum_{k=0}^{r\left(\xi^{\prime}\right)-1} \mathscr{B}_{k_{j}}^{\prime}\left(\xi^{\prime}\right)\left|\xi^{\prime}\right|^{-k} \delta_{k^{\prime} k} \\
& =\sum_{k^{\prime}=0}^{r\left(\xi^{\prime}\right)-1} \mathscr{B}_{j^{\prime} k^{\prime}}\left(\xi^{\prime}\right)\left|\xi^{\prime}\right|^{k^{\prime}} \sum_{j=0}^{p-1} \hat{g}_{j}\left(\xi^{\prime}\right) \mathscr{B}_{k^{\prime} j}^{\prime}\left(\xi^{\prime}\right)\left|\xi^{\prime}\right|^{-k^{\prime}} \\
& =\sum_{k^{\prime}=0}^{r\left(\xi^{\prime}\right)-1} \mathscr{B}_{j^{\prime} k^{\prime}}\left(\xi^{\prime}\right) \sum_{j=0}^{p-1} \mathscr{B}_{k^{\prime} j}\left(\xi^{\prime}\right) \hat{g}_{j}\left(\xi^{\prime}\right) \\
& =\sum_{j=0}^{p-1} \delta_{j^{\prime} j} \hat{g}_{j}\left(\xi^{\prime}\right) \\
& =\hat{g}_{j^{\prime}}\left(\xi^{\prime}\right) \text {, as required. }
\end{aligned}
$$

Conversely, suppose $B$ is right invertible. Since $B=\mathscr{B} \gamma$, we conclude $\mathscr{B}$ is also right invertible. Hence, by Lemma 2.2 , rank $\mathscr{B}\left(\xi^{\prime}\right)=p$ for all $\xi^{\prime} \neq 0$.

Suppose now that rank $\mathscr{B}\left(\xi^{\prime}\right)=r\left(\xi^{\prime}\right)$ for all $\xi^{\prime} \neq 0$. Then for these $\xi^{\prime}, \mathscr{B}\left(\xi^{\prime}\right)=$ $\left[\mathscr{B}_{1}\left(\xi^{\prime}\right) \mid 0\right]$ where $\mathscr{B}_{1}\left(\xi^{\prime}\right)$ is a $p \times r\left(\xi^{\prime}\right)$ matrix with left inverse $\mathscr{B}_{1}^{\prime}\left(\xi^{\prime}\right)=$ $\left(\mathscr{B}_{1}\left(\xi^{\prime}\right)^{*} \mathscr{B}_{1}\left(\xi^{\prime}\right)\right)^{-1} \mathscr{B}_{1}\left(\xi^{\prime}\right)^{*}$. Let $\mathscr{B}^{\prime}\left(\xi^{\prime}\right)$ denote the $r \times p$ matrix

$$
J_{2}\left(\xi^{\prime}\right)^{-1}\left[\frac{\mathscr{B}_{1}^{\prime}\left(\frac{\xi^{\prime}}{\left|\xi^{\prime}\right|}\right)}{0}\right] J_{1}\left(\xi^{\prime}\right)^{-1} \text {. }
$$

Setting $B^{\prime}=R Q \mathscr{B}^{\prime}$, we show that $B^{\prime} B=I$. 
For this, let $u \in Z_{\text {ker } A}^{s}\left(R_{+}^{n}\right)$ and set

$$
w=\left(D_{n}+i\left|\nabla^{\prime}\right|\right)^{s}\left(P u-Q \mathscr{B}^{\prime} \mathscr{B} \gamma P u\right)
$$

where $P$ is a suitable reflection operator. So $w \in L^{2}\left(R^{n}\right)$ and it suffices to prove that $w \in L^{2}\left(R_{-}^{n}\right)$. For then, $P u-Q^{\prime} \mathscr{B} P u \in Z^{s}\left(R_{-}^{n}\right)$ and $B^{\prime} B u=$ $R Q \mathscr{G}^{\prime} \mathscr{B} P u=R P u=u$.

Now $R A P u=0$, so $v=A P u \in Z^{s-m}\left(R_{-}^{n}\right)$. As $L^{2}\left(R_{-}^{n}\right)$ is dense in $Z^{s-m}\left(R_{-}^{n}\right)$, there exists $v_{i} \in L^{2}\left(R_{-}^{n}\right)$ with $v_{i} \rightarrow v$ in $Z^{s-m}\left(R_{-}^{n}\right)$. Then $R A^{-1} v_{i} \rightarrow u$ in $Z^{s}\left(R_{+}^{n}\right)$ and

$$
w_{i}=\left(D_{n}+i\left|\nabla^{\prime}\right|\right)^{s}\left(A^{-1} v_{i}-Q^{\prime} \mathscr{B} A^{-1} v_{i}\right) \rightarrow w \quad \text { in } L^{2}\left(R^{n}\right)
$$

Now for $\xi^{\prime} \neq 0$,

$$
\begin{aligned}
\hat{w}_{i}(\xi)=\left(\xi_{n}+i\left|\xi^{\prime}\right|\right)^{s}\left[A(\xi)^{-1} \hat{v}_{i}(\xi)-\left|\xi^{\prime}\right|^{h+m-1}\left(\xi_{n}+i\left|\xi^{\prime}\right|\right)^{-h} A(\xi)^{-1}\right. & \\
& \left.\cdot \sum_{j=0}^{r\left(\xi^{\prime}\right)-1}\left|\xi^{\prime}\right|^{-j} p_{j}\left(\frac{\xi^{\prime}}{\left|\xi^{\prime}\right|}, \frac{\xi_{n}}{\left|\xi^{\prime}\right|}\right) \int_{-\infty}^{\infty} \eta^{j} A\left(\xi^{\prime}, \eta\right)^{-1} \hat{v}_{i}\left(\xi^{\prime}, \eta\right) d \eta\right] .
\end{aligned}
$$

Take $\Gamma=\Gamma_{R}=[-R, R] \cup S_{R}$ where $S_{R}$ is the semi-circle $|z|=R, \operatorname{Im} z>0$, and $R$ is sufficiently large. Then

$$
\begin{aligned}
\left|\int_{S_{R}} z^{j} A\left(\xi^{\prime}, z\right)^{-1} \hat{v}_{i}\left(\xi^{\prime}, z\right) d z\right| & \leqslant c \int_{0}^{\pi} R^{j-m+1}\left|\hat{v}_{i}\left(\xi^{\prime}, R e^{i \theta}\right)\right| d \theta \\
& <c \int_{0}^{\pi} \int_{-\infty}^{0} R^{j-m+1}\left|e^{-i x_{n} R e^{n}} \tilde{v}_{i}\left(\xi^{\prime}, x_{n}\right)\right| d x_{n} d \theta \\
& =c \int_{0}^{\pi} \int_{-\infty}^{0} R^{j-m+1} e^{x_{n} R \sin \theta}\left|\tilde{v}_{i}\left(\xi^{\prime}, x_{n}\right)\right| d x_{n} d \theta
\end{aligned}
$$

$\tilde{v}_{i}\left(\xi^{\prime}, x_{n}\right)$ again denoting the partial Fourier transform of $v_{i}\left(x^{\prime}, x_{n}\right)$. Now since $0<j<r-1<m-1$, the integrand is dominated by the integrable function $\left|\tilde{v}_{i}\left(\xi^{\prime}, x_{n}\right)\right|$ on the region $G$ of integration. Moreover, as $R \rightarrow \infty$ the integrand converges pointwise almost everywhere on $G$ to zero. By Lebesgue's convergence theorem, the integral converges to zero as $R \rightarrow \infty$. Hence

$$
\begin{aligned}
\hat{w}_{i}(\xi)=\lim _{R \rightarrow \infty}\left(\xi_{n}+i\left|\xi^{\prime}\right|\right)^{s} A(\xi)^{-1}\left[\hat{v}_{i}(\xi)\right. & -\sum_{j=0}^{r\left(\xi^{\prime}\right)-1}\left(\frac{\xi_{n}}{\left|\xi^{\prime}\right|}+i\right)^{-h} p_{j}\left(\frac{\xi^{\prime}}{\left|\xi^{\prime}\right|}, \frac{\xi_{n}}{\left|\xi^{\prime}\right|}\right) \\
& \cdot \int_{\left.\Gamma_{R /\left|\xi^{\prime}\right|} A\left(\frac{\xi^{\prime}}{\left|\xi^{\prime}\right|}, \zeta\right)^{-1} \zeta^{j} \hat{v}_{i}\left(\xi^{\prime}, \zeta\left|\xi^{\prime}\right|\right) d \zeta\right] .} .
\end{aligned}
$$

By the Cauchy-Goursat theorem, this last integral is independent of $R$ for $R$ large enough. By (6.3), for almost every $\xi^{\prime}, \hat{w}_{i}(\xi)$ has an analytic extension to Im $\xi_{n}>0$. Setting $g(z)=\left(z+i\left|\xi^{\prime}\right|\right)^{-m} A\left(\xi^{\prime}, z\right)$ and $f(z)=g(z) \hat{w}_{i}\left(\xi^{\prime}, z\right)$, it follows 
that $f$ and $g$ satisfy the conditions of Lemma 6.4. So for almost every $\xi^{\prime}$, $\hat{w}_{i}\left(\xi^{\prime}, \xi_{n}\right) \in L^{2}\left(R_{-}^{1}\right)^{)}$. This means $w_{i} \in L^{2}\left(R_{-}^{n}\right)$ and hence so does $w$, as was required.

Conversely, suppose rank $\mathscr{B}\left(\eta^{\prime}\right)<r\left(\eta^{\prime}\right)$ for some $\eta^{\prime} \neq 0$. By Lemma $2.2 \mathscr{B}$ is not left invertible. Now if $r\left(\xi^{\prime}\right)$ is constant for $\xi^{\prime} \neq 0$ then, by what has been proved already, $\gamma=\left(\gamma_{0}, \ldots, \gamma_{r-1}\right)$ is an isomorphism. Hence $B=\mathscr{B}_{\gamma}$ is not left invertible.

So suppose $r\left(\xi^{\prime}\right)$ is not constant. Then $n=2$. Let $\psi \in L^{2}(R)$ with $\hat{\psi} \in$ $C_{0}^{\infty}(R), \hat{\psi}\left(\xi^{\prime}\right)=0$ for $\left|\xi^{\prime}\right|>\frac{1}{2}\left|\eta^{\prime}\right|$ and $\int\left|\hat{\psi}\left(\xi^{\prime}\right)\right|^{2} \mathrm{~d} \xi^{\prime}=1$. For $0<\varepsilon<1$, set

$$
\tilde{u}_{e}\left(\xi^{\prime}, x_{n}\right)=\varepsilon^{-(n-1) / 2}: \varepsilon^{-(n-1) / 2} \hat{\psi}\left(\frac{\xi^{\prime}-\eta^{\prime}}{\varepsilon}\right) \sum_{l=0}^{q} \sum_{s=0}^{m_{l}-1}\left(i x_{n}\right)^{s} e^{i x_{n}\left|\xi^{\prime}\right| z_{2}} g_{l_{s}}\left(\xi^{\prime}\right)
$$

where $z_{0}, \ldots, z_{q}$ are the distinct roots of $A^{+}\left(\eta^{\prime} /\left|\eta^{\prime}\right|, z\right)$ with multiplicities $m_{0}, \ldots, m_{q}$ and $g_{L s}\left(\xi^{\prime}\right)$ are continuous functions to be determined.

Now $\tilde{u}_{\varepsilon} \in L^{2}\left(R_{+}^{n}\right)$ and is therefore the partial Fourier transform of a function $u_{e} \in L^{2}\left(R_{+}^{n}\right)$. In fact $u_{\varepsilon} \in Z_{\text {ker } A}^{s}\left(R_{+}^{n}\right)$ for each real $s$. Furthermore,

$$
\begin{aligned}
D_{n}^{j} \tilde{u}_{e}\left(\xi^{\prime}, x_{n}\right)= & \varepsilon^{-(n-1) / 2}: \varepsilon^{-(n-1) / 2} \hat{\psi}\left(\frac{\xi^{\prime}-\eta^{\prime}}{\varepsilon}\right) \sum_{l=0}^{q} \sum_{s=0}^{m_{l}-1} \sum_{h=0}^{j}\left(\begin{array}{l}
j \\
h
\end{array}\right) \frac{s !}{(s-h) !}\left(i x_{n}\right)^{s-h} \\
& \cdot\left(z_{l}\left|\xi^{\prime}\right|\right)^{j-h} e^{i x_{n}\left|\xi^{\prime}\right| z_{l}} g_{l s}\left(\xi^{\prime}\right),
\end{aligned}
$$

and

$$
\left(\gamma_{j} u_{e}\right)^{-}\left(\xi^{\prime}\right)=\varepsilon^{-(n-1) / 2} \hat{\psi}\left(\frac{\xi^{\prime}-\eta^{\prime}}{\varepsilon}\right) \sum_{l=0}^{q} \sum_{s=0}^{m_{l}-1} \frac{j !}{(j-s) !}\left(z_{l}\left|\xi^{\prime}\right|\right)^{j-s} g_{l s}\left(\xi^{\prime}\right) .
$$

As before write $\mathscr{B}\left(\eta^{\prime}\right)=\left[\mathscr{B}_{1}\left(\eta^{\prime}\right) \mid 0\right]$ where $\mathscr{B}_{1}\left(\eta^{\prime}\right)$ is a $p \times r\left(\eta^{\prime}\right)$ matrix. Then rank $\mathscr{B}_{1}\left(\eta^{\prime}\right)<r\left(\eta^{\prime}\right)$ so there exists a unit vector $c=\left(c_{j}\right)$ such that $\mathscr{B}_{1}\left(\eta^{\prime}\right) c=0$. Consider the system of equations

$$
\left(\gamma_{j} u_{\varepsilon}\right)^{\wedge}\left(\xi^{\prime}\right)=\varepsilon^{-(n-1) / 2} \hat{\psi}\left(\frac{\xi^{\prime}-\eta^{\prime}}{\varepsilon}\right) c_{j}, \quad 0<j<r\left(\eta^{\prime}\right)-1 .
$$

There are $r\left(\eta^{\prime}\right)$ equations in the $r\left(\eta^{\prime}\right)$ unknowns $g_{l s}\left(\xi^{\prime}\right)$. Now the determinants

$$
\left|\frac{j !}{(j-s) !}\left(z_{l}\left|\xi^{\prime}\right|\right)^{j-s}\right|_{j,(l, s)}
$$

where $0<j \leqslant r\left(\eta^{\prime}\right)-1,0<l \leqslant q, 0<s<m_{l}-1$, has the value

$$
\prod_{l=0}^{q} \prod_{s=0}^{m_{l}-1}\left(s ! \prod_{h=0}^{l-1}\left(z_{l}\left|\xi^{\prime}\right|-z_{h}\left|\xi^{\prime}\right|\right)^{m_{h}}\right)
$$

which is nonzero. See Van der Poorten (1976). Hence the system (6.8) can be solved for functions $g_{t s}\left(\xi^{\prime}\right)$ continuous when $\xi^{\prime} \neq 0$. 
Finally,

$$
\begin{aligned}
& \left(B u_{e}\right)^{\wedge}\left(\xi^{\prime}\right)=\mathscr{B}\left(\xi^{\prime}\right)\left(\gamma u_{e}\right)^{\wedge}\left(\xi^{\prime}\right) \\
& =\mathscr{B}_{1}\left(\xi^{\prime}\right) \varepsilon^{-(n-1) / 2} \hat{\psi}\left(\frac{\xi^{\prime}-\eta^{\prime}}{\varepsilon}\right) c
\end{aligned}
$$

and it follows, as in the proof of Lemma 2.2, that $B u_{\varepsilon} \rightarrow 0$ as $\varepsilon \rightarrow 0$. However,

$$
\left\|\gamma u_{e}\right\|^{2}=\sum_{j=0}^{r\left(\eta^{\prime}\right)-1} \int_{-\infty}^{\infty}\left|\xi^{\prime}\right|^{2 s-2 j-1} \varepsilon^{-(n-1)}\left|\hat{\psi}\left(\frac{\xi^{\prime}-\eta^{\prime}}{\epsilon}\right)\right|^{2} c_{j}^{2} d \xi^{\prime} .
$$

Since the integrand is zero outside the interval $\eta^{\prime}-\frac{1}{2}\left|\eta^{\prime}\right|<\xi^{\prime}<\eta^{\prime}+\frac{1}{2}\left|\eta^{\prime}\right|$ we conclude that

$$
\left\|\gamma u_{\varepsilon}\right\|^{2}>\kappa \sum_{j=0}^{r\left(\eta^{\prime}\right)-1} \int_{-\infty}^{\infty} \varepsilon^{-(n-1)}\left|\hat{\psi}\left(\frac{\xi^{\prime}-\eta^{\prime}}{\varepsilon}\right)\right|^{2} c_{j}^{2} d \xi^{\prime}=\kappa>0 .
$$

So $\left\|\gamma u_{e}\right\|$ and $\left\|u_{e}\right\|$ are uniformly bounded away from zero, yet $B u_{\varepsilon} \rightarrow 0$. Hence $B$ is not left invertible.

COROLlaRy 6.9. Let $A$ be an elliptic operator of the form (1.1) and $s$ real. Then

$$
\left(\gamma_{0}, \ldots, \gamma_{p-1}\right): Z_{\mathrm{ker} A}^{s}\left(R_{+}^{n}\right) \rightarrow \prod_{j=0}^{p-1} Z^{s-j-(1 / 2)}\left(R^{n-1}\right)
$$

is left (right) invertible if and only if $p>r\left(\xi^{\prime}\right)\left(\right.$ respectively $p<r\left(\xi^{\prime}\right)$ ) for all $\xi^{\prime} \neq 0$.

Proof. When $p>r\left(\xi^{\prime}\right), \mathscr{B}\left(\xi^{\prime}\right)=\left[\begin{array}{l}I \\ 0 \\ 0\end{array}\right]$ where $I$ is the $r\left(\xi^{\prime}\right) \times r\left(\xi^{\prime}\right)$ identity matrix. When $p<r\left(\xi^{\prime}\right), \mathscr{B}\left(\xi^{\prime}\right)=\left[\begin{array}{ll}I & 0\end{array}\right]$ where $I$ is now the $p \times p$ identity. The result now follows.

Corollary 6.10. Let $A$ be an elliptic operator of the form (1.1), B a boundary system of the form (1.2), and $s>\max \left(m_{j}+\frac{1}{2}\right)$. Then

$$
(A, B): Z^{s}\left(R_{+}^{n}\right) \rightarrow Z^{s-m}\left(R_{+}^{n}\right) \times \prod_{j=0}^{p-1} Z^{s-m-(1 / 2)}\left(R^{n-1}\right)
$$

is left (right) invertible if and only if rank $\mathscr{B}^{\prime}\left(\xi^{\prime}\right)=r\left(\xi^{\prime}\right)$ (respectively $p$ ) for all $\xi^{\prime} \neq 0$.

Proof. By Theorem 2.1,A: $Z^{s}\left(R_{+}^{n}\right) \rightarrow Z^{s-m}\left(R_{+}^{n}\right)$ is right invertible. By application (4.2) of the five lemma of Pryde (1977), $(A, B)$ is left (right) invertible if and only if $B \mid$ ker $A$ is left (respectively right) invertible. 


\section{A priori inequalities in Sobolev spaces}

We use the results of the previous sections to obtain a priori inequalities for boundary value problems in Sobolev spaces. For the case when $A(\xi)$ is of even order similar results were obtained by Schechter (1959), Agmon-DouglisNirenberg (1959), Browder (1959), and others.

Let $A$ be an operator whose symbol is of the form

$$
A(\xi)=\sum_{k=0}^{m} \sum_{j=0}^{k} a_{j}^{k}\left(\xi^{\prime}\right) \xi_{n}^{j}
$$

where the $a_{j}^{k}\left(\xi^{\prime}\right)$ are positively homogeneous of order $k-j$ and are continuous for $\xi^{\prime} \neq 0$.

Then $A$ is called elliptic if its principal symbol $A^{m}(\xi)=\sum_{j=0}^{m} a_{j}^{m}\left(\xi^{\prime}\right) \xi_{n}^{j}$ is non-vanishing for real $\xi \neq 0$. Further, $A$ is called homogeneous if $A(\xi)=A^{m}(\xi)$.

Let $B=\left(B_{j}\right), 0 \leqslant j \leqslant p-1$, be a boundary system with symbol of the form

$$
B_{j}(\xi)=\sum_{l=0}^{m_{j}} B_{j}^{\prime}(\xi)
$$

where, for $l<m_{j}, B_{j}^{l}(\xi)$ is positively homogeneous of order $l$ and continuous for $\xi \neq 0$, and $B_{j}^{m^{\prime}}(\xi)=\sum_{k=0}^{m_{j}} b_{j k}\left(\xi^{\prime}\right) \xi_{n}^{k}$, with $b_{j k}\left(\xi^{\prime}\right)$ positively homogeneous of order $m_{j}-k$ and continuous for $\xi^{\prime} \neq 0$.

Again, $B$ is called homogeneous if $\left(B_{j}(\xi)\right)=\left(B_{j}^{m}(\xi)\right)$.

If $A$ is elliptic, we can construct the matrix $\mathscr{B}\left(\xi^{\prime}\right)$ and the numbers $r\left(\xi^{\prime}\right)$ from $A^{m}(\xi)$ and $\left(B_{j}^{m}(\xi)\right)$ as in Section 5.

Let $Q=\left\{x \in R^{n}:|x|<1\right\}$ and $Q_{+}=\left\{x \in Q: x_{n}>0\right\}$.

THEOREM 7.3. If $A$ is an operator of the form (7.1) and $s$ is real, the following are equivalent in $\bar{Q}$;

(a) $\|u\|_{H^{s}\left(R^{n}\right)} \leqslant c\left(\|A u\|_{H^{s-m_{\left(R^{n}\right)}}}+\|u\|_{H^{s-1}\left(R^{n}\right)}\right.$ for all $u \in H^{s}\left(R^{n}\right)$ with support

(b) $A$ is elliptic.

THEOREM 7.4. If $A$ is a homogeneous elliptic operator of the form (7.1), B is a boundary system of the form (7.2) and $s$ is real, the following are equivalent: $\bar{Q}_{+}$;

(a) $\|u\|_{H^{\prime}\left(R_{+}^{n}\right)} \leqslant c\left(\|B u\|_{H}+\|u\|_{H^{s-1}\left(R_{+}^{n}\right)}\right)$ for all $u \in H_{\text {ker } A}^{s}\left(R_{+}^{n}\right)$ with support in

(b) rank $\mathscr{B}\left(\xi^{\prime}\right)=r\left(\xi^{\prime}\right)$ for all $\xi^{\prime} \neq 0$. 
THEOREM 7.5. If $A$ is an elliptic operator of the form (7.1), $B$ is a boundary system of the form (7.2) and $s>\max \left(m_{j}+\frac{1}{2}\right)$, the following are equivalent:

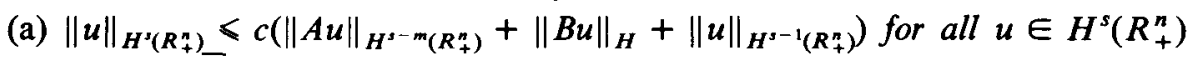
with support in $\bar{Q}_{+}$;

(b) rank $\mathscr{B}\left(\xi^{\prime}\right)=r\left(\xi^{\prime}\right)$ for all $\xi^{\prime} \neq 0$.

Here, $\|B u\|_{H}$ denotes the norm of $B u$ in $\prod_{j=0}^{p-1} H^{s-m_{-}-(1 / 2)}\left(R^{n-1}\right)$. These three theorems follow respectively from Theorem 2.1, Theorem 6.5 , and Corollary 6.10. We give the details for one case only.

Proof of Theorem 7.4. Suppose (a) holds. For suitably large integer $l$, let $u \in C_{0}^{l}\left(\overline{R_{+}^{n}}\right) \cap Z_{\text {ker } A}^{s}\left(R_{+}^{n}\right)$, which by Theorem 3.1 is dense in $Z_{\text {ker } A}^{s}\left(R_{+}^{n}\right)$. Define $u_{\varepsilon}(x)=\varepsilon^{s-(n / 2)} u(x / \varepsilon)$. Then there exists $\varepsilon_{0}>0$ such that $u_{\varepsilon} \in C_{0}^{l}\left(\bar{Q}_{+}\right)$if $0<\varepsilon$ $<\varepsilon_{0}$. As $\varepsilon \rightarrow 0,\left\|u_{\varepsilon}\right\|_{H^{s}\left(R_{7}^{\eta}\right)} \rightarrow\|u\|_{Z^{s}\left(R_{+}^{n}\right)},\left\|B u_{\varepsilon}\right\|_{H} \rightarrow\left\|\left(B_{j}^{m_{j}} u\right)\right\|_{Z}$, where $Z=$ $\Pi_{j=0}^{p-1} Z^{s-m_{j}-(1 / 2)}\left(R^{n-1}\right)$, and $\left\|u_{\varepsilon}\right\|_{H^{s-1}\left(R_{+}^{n}\right)} \rightarrow 0$. Hence

$\left(\mathrm{a}^{\prime}\right)\|u\|_{Z^{x^{\prime}\left(R_{+}^{n}\right)}} \leqslant c\left\|\left(B_{j}^{m} u\right)\right\|_{Z}$ for all $u \in Z_{\mathrm{ker} A}^{s}\left(R_{+}^{n}\right)$.

So $\left(B_{j}^{m^{\prime}}\right)$ is left invertible and by Theorem 6.5 rank $\mathscr{B}\left(\xi^{\prime}\right)=r\left(\xi^{\prime}\right)$ for all $\xi^{\prime} \neq 0$.

Conversely, if rank $\mathscr{B}\left(\xi^{\prime}\right)=r\left(\xi^{\prime}\right)$ for $\xi^{\prime} \neq 0$, then inequality $\left(a^{\prime}\right)$ holds. In particular (a') holds for all $u \in C_{0}^{l}\left(\bar{Q}_{+}\right) \cap Z_{\mathrm{ker} A}^{s}\left(R_{+}^{n}\right)$. But the Sobolev and homogeneous norms are equivalent on this space (Pryde (1980a), Theorem 6.1) and so

$\left(\mathrm{a}^{\prime \prime}\right)\|u\|_{H^{s}\left(R_{+}^{n}\right)} \leqslant c \|\left(B_{j}^{\left.m^{\prime} u\right) \|_{H}} \leqslant c\left(\|B u\|_{H}+\|u\|_{\left.H^{s-i_{(}\left(_{+}^{n}\right)}\right)}\right)\right.$.

Finally, the closure of $C_{0}^{l}\left(\bar{Q}_{+}\right) \cap Z_{\mathrm{ker} A}^{s}\left(R_{+}^{n}\right)$ in $H^{s}\left(R_{+}^{n}\right)$ has finite codimension in $\left\{u \in H^{s}\left(R_{+}^{n}\right)\right.$ : support $\left.u \subset \bar{Q}_{+}\right\}$. On any complement of that closure, $\|u\|_{H^{\prime}\left(R_{+}^{n}\right)} \sim\|u\|_{H^{s-1}\left(R_{+}^{n}\right)}$. Hence inequality (a) holds.

\section{Regularity results}

THEOREM 8.1. Let $A$ be an elliptic operator of the form (7.1) and $s$ real.

(a) If $u \in L^{2}\left(R^{n}\right)$ and $A u \in H^{s-m}\left(R^{n}\right)$ then $u \in H^{s}\left(R^{n}\right)$.

(b) If $A$ is homogeneous with $u \in L^{2}\left(R^{n}\right)$ and $A u \in Z^{s-m}\left(R^{n}\right)$ then $u \in$ $H^{s}\left(R^{n}\right)$.

PRoof. (b) Since $A$ is homogeneous and elliptic, $\|u\|_{Z^{\prime}\left(R^{n}\right)} \sim\|A u\|_{Z^{*-m_{\left(R^{n}\right)}}}$ by Theorem 2.1. Hence $u \in Z^{s}\left(R^{n}\right) \cap L^{2}\left(R^{n}\right) \subset H^{s}\left(R^{n}\right)$.

(a) Write $A=A^{m}+A^{0}$ where $A^{0}$ here denotes the lower order part of $A$. The proof is by induction on $s$. Firstly, the result is trivial for $s<0$. Suppose it holds for $s<k$ where $k$ is a non-negative integer and consider $k<s<k+1$. By part 
(b) it suffices to prove that $A^{m} u \in Z^{s-m}\left(R^{n}\right)$. Now $A u \in H^{s-m}\left(R^{n}\right) \subset$ $H^{k-m}\left(R^{n}\right)$ and so, by the induction hypothesis, $u \in H^{k}\left(R^{n}\right)$. So $A^{0} u \in$ $H^{k-m+1}\left(R^{n}\right) \subset H^{s-m}\left(R^{n}\right)$ and $A^{m} u=A u-A^{0} u \in H^{s-m}\left(R^{n}\right) \cap Z^{-m}\left(R^{n}\right)$. But for positive $m$ and $s$ there exists $c$ such that

$$
|\xi|^{s-m}<c\left[\left(1+|\xi|^{2}\right)^{(s-m) / 2}+|\xi|^{-m}\right] \text { for all } \xi .
$$

Hence $A^{m} u \in Z^{s-m}\left(R^{n}\right)$ as required.

THEORem 8.2. Let $A$ be an elliptic operator of the form (7.1) and $B$ a boundary system of the form (7.2) with rank $\mathscr{B}\left(\xi^{\prime}\right)=r\left(\xi^{\prime}\right)$ for $\xi^{\prime} \neq 0$. Let $s$ be real and $t>\max \left(m_{j}+\frac{1}{2}\right)$.

(a) If $u \in H^{t}\left(R_{+}^{n}\right), A u \in H^{s-m}\left(R_{+}^{n}\right)$ and $B u \in \Pi_{j=0}^{p-1} H^{s-m-(1 / 2)}\left(R^{n-1}\right)$ then $u \in H^{s}\left(R_{+}^{n}\right)$.

(b) If $A$ and $B$ are homogeneous with $u \in H^{t}\left(R_{+}^{n}\right), A u \in Z^{s-m}\left(R_{+}^{n}\right)$ and $B u \in \Pi_{j=0}^{p-1} Z^{s-m_{j}-(1 / 2)}\left(R^{n-1}\right)$ then $u \in H^{s}\left(R_{+}^{n}\right)$.

(c) If $A$ and $B$ are homogeneous with $u \in L^{2}\left(R_{+}^{n}\right), A u=0$ and $B u \in$ $\Pi_{j=0}^{p-1} Z^{s-m_{j}-(1 / 2)}\left(R^{n-1}\right)$ then $u \in H^{s}\left(R_{+}^{n}\right)$.

Proof. (c) Since rank $\mathscr{B}\left(\xi^{\prime}\right)=r\left(\xi^{\prime}\right)$ for $\xi^{\prime} \neq 0, \quad B: L_{\text {ker } A}^{2}\left(R_{+}^{n}\right) \rightarrow$ $\prod_{j=0}^{p-1} Z^{-m_{j}-(1 / 2)}\left(R^{n-1}\right)$ has a bounded left inverse $B^{\prime}$ (Theorem 6.5). By taking the constant $h$, in the construction given for $B^{\prime}$, large enough, we can ensure that $B^{\prime}$ extends to a bounded operator $B^{\prime}: \Pi_{j=0}^{p-1} Z^{s-m_{j}-(1 / 2)}\left(R^{n-1}\right) \rightarrow Z_{\text {ker } A}^{s}\left(R_{+}^{n}\right)$. So $u=B^{\prime}(B u) \in Z_{\text {ker } A}^{s}\left(R_{+}^{n}\right) \cap L^{2}\left(R_{+}^{n}\right) \subset H_{\text {ker } A}^{s}\left(R_{+}^{n}\right)$.

(b) Let $P$ be a suitable reflection operator and set $v=u-R A^{-1} P A u$. Since $u \in H^{t}\left(R_{+}^{n}\right)$, by Lemma 3.6, $v \in H^{t}\left(R_{+}^{n}\right)$. Moreover, $B v=B u-B R A^{-1} P A u \in$

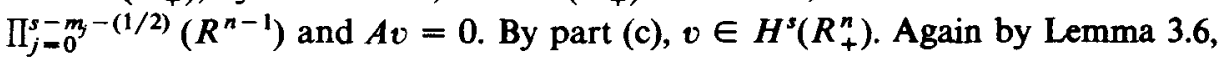
$R A^{-1} P A u \in H^{\prime}\left(R_{+}^{n}\right) \cap Z^{s}\left(R_{+}^{n}\right) \subset H^{s}\left(R_{+}^{n}\right)$. So $u \in H^{s}\left(R_{+}^{n}\right)$.

(a) This is proved by induction on $s$ using part (b) as in the proof of the previous theorem.

\section{References}

S. Agmon, A. Douglis and L. Nirenberg (1959), 'Estimates near the boundary for solutions of elliptic partial differential equations satisfying general boundary conditions, I', Comm. Pure Appl. Math. 12, 623-727.

F. E. Browder (1959), 'Estimates and existence theorems for elliptic boundary problems', Proc. Nat. Acad. Sci. U.S.A. 45, 365-372.

A. J. Pryde (1977), 'The five lemma for Banach spaces', Proc. Amer. Math. Soc. 65, 37-43.

A. J. Pryde (1980a), 'Spaces with homogeneous norms', Bull. Austral. Math. Soc. 21, 189-205. 
A. J. Pryde (1980b), 'Second order elliptic boundary value problems in spaces with homogeneous norms', J. Austral. Math. Soc. Ser. A 29, 1-13.

A. J. Pryde (1981), 'Second order elliptic equations with mixed boundary conditions', J. Math. Anal. Appl., to appear.

M. Schecter (1959), 'Integral inequalities for partial differential operators and functions satisfying general boundary conditions', Comm. Pure Appl. Math. 12, 37-66.

A. J. Van der Poorten (1976), 'Some determinants that should be better known', J. Austral. Math. Soc. Ser. A 21, 278-288.

Department of Mathematics

University of Toronto

Toronto

Canada 